\title{
Zukunftslos aktiviert oder zukunftsfähig investiert?
}

\section{Olaf Struck, Susanne Gerstenberg, Alexandra Krause, Ina Krause}

Auf dem deutschen Arbeitsmarkt nehmen Flexibilität und Mobilität zu. Ob sich daraus soziale Chancen oder Risiken ergeben, hängt nicht zuletzt von der Ausgestaltung der Sozial- und Arbeitsmarktpolitik ab. Der Beitrag sieht in der aktuellen, auf kurzfristige Kosteneffizienz zielenden Aktivierungsstrategie keine Chancen. Mittelfristig Erfolg versprechend sind jedoch Maßnahmen, die Sicherheiten für Nichterwerbsphasen bieten, Übergangsrisiken mindern, Investitionen in den Bereichen Bildung, Beruf und Familie fördern und differenzierte Kompetenzprofile der Individuen berücksichtigen. So können der Arbeitsmarktzugang und die Bewältigung der wachsenden Flexibilitäts- und Qualifikationsanforderungen verbessert werden.

\section{Die Ausgangssituation}

Seit den 1990er Jahren steigt die Mobilität am Arbeitsmarkt (Struck 2006; Struck et al. 2007). Damit verbunden werden Berufsund Lebensläufe individueller und unsicherer. Zugleich bleiben sie abhängig von Institutionen wie Arbeitsmarkt, Bildungsund sozialen Sicherungssystemen (Beck/ Beck-Gernsheim 1993). Das heißt, es gibt eine Abhängigkeit der Lebenslaufgestaltung von institutionellen Vorgaben. Letztere stellen folglich eine indirekte und „weiche“ Beeinflussung dar (Allmendinger 1994; Kaufmann 1986). Mit steigender Mobilität sind die Anforderungen an die Individuen gestiegen, die eigene Erwerbsplanung immer wieder neu zu justieren. Im fortwährenden Prozess der Planungen zur Erreichung ihrer Ziele und Wünsche sind die Individuen jedoch mit dem Problem konfrontiert, zum Teil widersprüchliche institutionelle Anforderungen in Einklang zu bringen.

Von besonderer Bedeutung sind in der deutschen Gesellschaft u. a. die unzulängliche Abstimmung der Mobilitäts- und Flexibilisierungspraxis am Arbeitsmarkt mit den Normalarbeitsunterstellungen der sozialen Sicherungssysteme. Beschäftigungsrisiken werden auf diese Weise tendenziell individualisiert. Diese Situation wird im ersten Teil des Beitrages dargestellt (Abschnitt 1.1-1.3). Im zweiten Teil (Abschnitt 2) werden dann arbeitsmarkt- und sozialpolitische Gestaltungsoptionen skizziert, die geeignet sind, Individuen ausreichend gegen soziale Risiken abzusichern und zugleich ihre individuellen Kompetenzen zur Bearbeitung von Flexibilitätsanforderungen zu steigern, dabei aber auch dem betrieblichen Bedarf an Anpassungsflexibilität gerecht zu werden und den Arbeitsmarkt zu entlasten. Abschnitt 3 zieht ein resümierendes Fazit.

\subsection{FLEXIBILITÄTSANFORDERUNGEN AM ARBEITSMARKT}

In der deutschen Nachkriegsgeschichte war die Erzielung von Leistungsbereitschaft lange Zeit an Sicherheitsversprechen seitens der Arbeitgeber gekoppelt. Stabilisiert wurde dieses Austauscharrangement durch Institutionen der Berufsfachlichkeit und kollektiven Lohnfindung, die Investitionen in berufliche Qualifikation, Einkommensstabilität und ein Existenz sicherndes Lohnniveau förderten. Im internationalen Vergleich waren Betriebswechsel selten, beschränkten sich zumeist auf berufliche Einstiegsphasen und waren in der Regel mit einem beruflichen Aufstieg verbunden. Diskontinuitäten auf Absatzmärkten wurden von Unternehmen vorrangig intern über flexible Arbeitszeiten (Überstunden, Teilzeit, Arbeitszeitkonten, Kurzarbeit) und teilweise über Prämienlöhne ausgeglichen. Zwar war die Situation der Beschäftigten auch in dieser Zeit der Prosperität an Marktanpassungsprozesse der Unternehmen gekoppelt, jedoch konnten sie Arbeitsplatzsicherheit erwarten und ihre In- vestitionen in den Bereichen Bildung, Beruf und Familie etc. daran ausrichten.

Doch mehr und mehr kommt es zu Auflösungen der impliziten und der tarifvertraglich oft auch explizit geregelten Kontrakte „Sicherheit gegen Leistung“. Mit der Ausweitung der Finanzmärkte und der Öffnung von Märkten in Asien und Osteuropa gewannen sowohl kurzfristige als auch höhere Gewinnerwartungen der nunmehr global flexibleren Kapitalgeber an Bedeutung. Auf externes Kapital angewiesene

Olaf Struck, Prof. Dr., lehrt Arbeitswissenschaft an der Universität Bamberg und ist Projektleiter im Sonderforschungsbereich 580 der Universitäten Jena und Halle. e-mail: olat.struck@uni-bamberg.de Susanne Gerstenberg, wissenschaftliche Mitarbeiterin im SFB 580 der Universitäten Jena und Halle. Arbeitsschwerpunkte: Quantitative Arbeitsmarkt- und Sozialstrukturanalyse.

e-mail: susanne.gerstenberg@uni-jena.de Alexandra Krause, Dr., wissenschaftliche Mitarbeiterin am Institut für Soziologie der Universität Jena und Projektleiterin im SFB 580 der Universitäten Jena und Halle. Arbeitsschwerpunkte: Quantitative Arbeitsmarkt- und Sozialstrukturanalyse. e-mail: alexandra.krause@uni-jena.de Ina Krause, wissenschaftliche Mitarbeiterin im SFB 580 der Universitäten Jena und Halle. Arbeitsschwerpunkte: Quantitative Arbeitsmarkt- und Transformationsforschung. e-mail: ina.krause@uni-jena.de 
Unternehmen müssen ihre Gewinnerwartungen gegenüber Kapitalgebern glaubhaft offenlegen. Systematischer als zuvor versuchen sie, u.a. durch Benchmarking und die Einführung von Cost-Centern, Transparenz zu steigern. Zudem begegnen sie dem Konkurrenz- und Innovationsdruck vielfach durch externe Flexibilitätsmaßnahmen wie z. B. Holdingbildung, Flexibilisierung der Unternehmensorganisation, Verringerung der Fertigungstiefe, Outsourcing, Offshoring und numerische Beschäftigungsanpassungen. Zugleich wird die interne Flexibilität erhöht: Dies u.a. durch Führung über Gewinnmargen und Zielvereinbarungen oder Arbeitszeit- und Lohnflexibilität.

Im alltäglichen Gefecht der Unternehmen um Kapitalgeber und die Erfüllung von Gewinnerwartungen haben sich die Aushandlungsergebnisse zuungunsten der Beschäftigten verschoben. Hinzu kommt eine hohe Arbeitslosigkeit, die Beschäftigungsrisiken bei Betriebsaustritten - sei es durch Eigenkündigung oder von Seiten des Betriebes - deutlich erhöht. Ausgestattet mit derart neuen Optionen und häufig selbst unter Druck, brechen viele Manager die tradierten Kontrakte von Arbeitsplatzund Lohnsicherheit gegen Leistung. Stellenabbau zur Steigerung einer häufig ohnehin hohen Kapitalrendite, Austausch gering Qualifizierter gegen höher Qualifizierte, der Einsatz zeitflexibler Vertragsformen wie Zeitarbeit und geringfügige Beschäftigung erhöhen die externe Flexibilität. Höhere Qualifikationsanforderungen, Leistungsverdichtung, Lohnsenkungen, die Absenkung des Krankenstandes und Angriffe auf bestehende rechtliche Schutzmechanismen wiederum steigern die interne Flexibilität. Externe und interne Flexibilisierung geben Marktrisiken auf zweifache Weise an die Belegschaften weiter, und Marktanpassungsprozesse von Unternehmen erfolgen deutlich stärker als zuvor unter den Bedingungen von Beschäftigungsunsicherheit.

Dies wird umso offenkundiger, als sich Beschäftigungsaustausche nicht mehr auf Berufseinsteiger und die gering qualifizierten Ränder der Unternehmen beschränken, sondern auch mittlere und ältere Altersgruppen und dabei auch qualifizierte Beschäftigte einbeziehen. Unsere Untersuchungen zur Entwicklung von Beschäftigungsstabilität (Struck et al. 2007; Köhler et al. 2008) zeigen (bei Kontrolle soziodemografischer und konjunktureller Ein- flussfaktoren) einen Rückgang der betrieblichen Verweildauern seit Mitte der 1990er Jahre. Immer schon bestehen hohe Austrittsraten in den ersten Jahren nach Betriebseintritt. Jedoch werden zunehmend auch Beschäftigungsverhältnisse mit mittlerer und höherer Betriebszugehörigkeitsdauer beendet.

Ausnahmen bestehen für zwei Gruppen. Zum einen sind Beschäftigte mit betriebsspezifischen Qualifikationen immer noch relativ sicher beschäftigt. Allerdings ist der Anteil der „unentbehrlichen“ betriebsspezifisch qualifizierten Beschäftigten gering. Dies ist insofern bedeutsam, da in der Arbeitsmarkttheorie, wie etwa in Segmentations- oder Transaktionskostenansätzen (Sengenberger 1987; Williamson et al. 1975), betriebsspezifische Qualifikationen als eine zentrale Entstehungsbedingung interner und stabiler Arbeitsmärkte gelten. In unserer Befragung von Personalverantwortlichen geben diese an, dass heute (lediglich) $20 \%$ ihrer Beschäftigten betriebsspezifische Qualifikationen besitzen, die nur selten in anderen Unternehmen benötigt würden. Diese Aussagen beziehen sich vor allem auf die klassischen Bereiche stabiler Beschäftigung Chemie (38 \%) und Maschinenbau (30\%) (Struck et al. 2007; Struck 2006, S. 187ff.). Zum anderen weisen von jenen Beschäftigten mit höheren allgemeinen Qualifikationen Personen in mittleren und höheren Steuerungs- und Führungsfunktionen vergleichsweise geringe Anteile an Betriebsaustritten auf. Sie haben qualitäts- und effizienzsichernde und im flexibleren Unternehmen zugleich stabilisierend wirkende soziale und kulturelle Funktionen zu erfüllen.

Mit der Ausweitung von Dienstleistungstätigkeiten, der Verkürzung von Innovationszyklen und dem damit verbundenen Bedeutungsgewinn allgemeiner und übertragbarer Qualifikationen haben also selbst berufsfachlich und höher Qualifizierte eine wichtige Basis ihrer „Unersetzlichkeit" verloren. Arbeit wird mobiler und flexibler. Berufsbilder verändern sich und Qualifikationsanforderungen steigen fortlaufend. Individuen sind daher mit einer höheren Notwendigkeit konfrontiert, in Beruf und Bildung zu investieren. Diese Investitionen werden zugleich aber auch unsicherer. Eine solche Entwicklung verlangt nach Lösungen für die Gestaltung von Flexibilität und Mobilität, Lösungen, die individuelle Flexibilität auf effiziente Weise fördern und zugleich Polarisierungen zwi- schen Insidern und Outsidern vermeiden. Die bisherige Arbeitsmarkt- und Sozialpolitik wird diesen neuen Anforderungen noch nicht gerecht.

\subsection{FUNKTIONSBEDINGUNGEN BESTEHENDER SOZIALER SICHERUNGSSYSTEME}

Systeme sozialer Sicherheit sind in national spezifischer Weise an Arbeitsmärkte und damit an Einkommen durch Erwerbsarbeit gekoppelt und weisen charakteristische, von Interessengruppen auch unter wechselseitig akzeptierten gesamtgesellschaftlichen Effizienzkriterien ausgehandelte Finanzierungs-, Auszahlungs- und Legitimationsmuster auf. Die zuvor aufgezeigten ökonomischen und sozialen Veränderungen stellen jedoch bestehende Verkopplungen des Erwerbsarbeits- und des sozialen Sicherungssystems infrage.

Die bisherige Kopplung ist in Deutschland institutionell durch das Normalarbeitsverhältnis ${ }^{1}$ und die Lohnarbeitszentriertheit der sozialen Sicherungssysteme geprägt (Vobruba 1990; Struck 2006). Beide Merkmale ergänzen sich und erzeugen Stabilität sowohl durch Finanztransfers in Form von Lohnsteuern und Sozialversicherungsabgaben als auch durch die Aufrechterhaltung bzw. (Wieder-)Herstellung der Arbeitsfähigkeit und -bereitschaft (aktive Unterstützung von Aus- und Weiterbildung, gesundheitliche Rehabilitation etc.) sowie die Sicherung von Investitionsentscheidungen für Arbeitnehmer und Arbeitgeber (Bildungs-, Arbeitsund Berufsstandards etc.). Dabei ist der Transfer von Sozialleistungen an drei lohnarbeitszentrierte Prinzipien geknüpft: Anwartschaft durch Lohnarbeit(szeiten), (Status-)Äquivalenz durch Anspruchsgewährung entsprechend der vorherigen Lohnhöhe und Arbeitsbereitschaft (Vobruba 1990, S. 28f.).

Eine solche Kopplung zwischen Erwerbsarbeit und sozialem Sicherungssystem ist voraussetzungsvoll: Erstens muss ausreichend Erwerbsarbeit für alle Erwerbspersonen vorhanden sein, die sich nicht in gesellschaftlich akzeptierten Nicht-Er-

\footnotetext{
1 Mückenberger (1985) definiert Normalarbeitsverhältnisse als zeitlich stabile und mindestens Existenz sichernd entlohnte, abhängige Vollzeittätigkeiten, deren Rahmenbedingungen arbeits- und sozialrechtlich geregelt sind.
} 
werbsphasen befinden (z. B. Bildungs-, Krankheits- oder Pflegephasen). Zweitens müssen Erwerbseinkommen ein Existenz sicherndes Mindesteinkommen überschreiten, damit die Finanzierung von Ansprüchen auf Sozialversicherungsleistungen etwa im Rentenalter oder Krankheitsfall über abhängige Erwerbsarbeit gewährleistet ist. Und drittens sind jene, die eine Stelle suchen, aktiv bei der Überwindung von Problemen zu fördern, die eine Aufnahme einer längerfristig Existenz sichernden Arbeit verhindern.

\subsection{DAS PROBLEM DER MANGELNDEN KOPPLUNG}

Keine der drei Funktionsvoraussetzungen ist derzeit erfüllt. Angesichts von jahresdurchschnittlich 3,3 Mio. registrierten Arbeitslosen $(7,8 \%)$, darunter die Hälfte Langzeitarbeitslose, sind selbst Ende der konjunkturellen Aufschwungsphase 2008 ausreichende Beschäftigungschancen nicht gegeben.

Im Niedriglohnbereich bleibt die Erlangung individueller Anwartschaften auf sozialen Schutz bei Arbeitslosigkeit, Krankheit und Rente prekär. Das stellt die Finanzierung der sozialen Sicherung insgesamt infrage. Von allen abhängig Beschäftigten waren 2007 gut $22 \%$ zu Niedriglöhnen (unterhalb von zwei Dritteln des Medians) tätig. Gut zwei Drittel der Betroffenen haben eine abgeschlossene Berufsausbildung (Kalina/Weinkopf 2009).

Auch bei den flexiblen Beschäftigungsformen, die vom Normalarbeitsverhältnis abweichen und vielfach nicht Existenz sichernd sind, droht die Verbindung zwischen Erwerbsarbeit und den normalarbeitsfixierten und lohnarbeitszentrierten sozialen Sicherungssystemen zu zerreißen. Von den 39,7 Mio. Erwerbstätigen im Jahr 2007 waren 26,9 Mio. (67,7 \%) sozialversicherungspflichtig beschäftigt. 6,9 Mio. Menschen (17,4 \%) waren in geringfügig entlohnter Beschäftigung tätig, darunter etwa zwei Drittel Frauen. $20 \%$ der Erwerbstätigen, darunter wiederum weit überwiegend Frauen, arbeiteten Teilzeit (Sachverständigenrat 2007). Etwa 2,4 \% der Beschäftigten sind Zeitarbeitnehmer (Bundesagentur für Arbeit 2008).

Die Ausbreitung flexibler Beschäftigungsformen wäre grundsätzlich ebenso wenig problematisch wie eine prinzipiell durchaus wünschenswerte Zunahme flexibler Erwerbsverläufe. Problematisch ist jedoch, dass Arbeitslosigkeit, mangelnde Arbeitsmarktchancen nach Beendigung eines Beschäftigungsverhältnisses und eine steigende Anzahl zeitflexibler Beschäftigungsverhältnisse die vom System sozialer Sicherungen gegenwärtig noch vorausgesetzte Stabilität und Kontinuität beruflicher Verläufe für einen steigenden Anteil der Beschäftigten vermissen lassen.

Neben den fast gänzlich Chancenlosen, die einen erheblichen Teil der Langzeitarbeitslosen ausmachen, wechseln viele Beschäftigte zwischen kurzen Phasen von (befristeter) Erwerbsarbeit, Familien- und Pflegephasen, Arbeitslosigkeit und arbeitsmarktpolitischen Maßnahmen. Alda (2006) zeigt, dass eine solche Situation - je nach Indikator - in Westdeutschland auf etwa $10 \%$ bis $15 \%$ und in Ostdeutschland auf etwa $27 \%$ bis $35 \%$ zutrifft. Frauen sind von einer solchen prekären Situation häufiger betroffen als Männer, Ältere etwas häufiger als Jüngere.

Im Westen fallen vor allem Geringqualifizierte in diesen Personenkreis, im Osten zum Teil Gering-, aber auch Fehlqualifizierte. Darunter finden sich dann junge Menschen - häufig mit Migrationshintergrund -, die mit hoher Wahrscheinlichkeit keinen Zugang zu einer stabilen und auskömmlichen Beschäftigung erhalten werden. Als Risikogruppen können zudem (alleinerziehende) Frauen mit Kindern sowie Erwerbspersonen im mittleren und höheren Alter gelten, die nach einer Phase ohne Erwerbsarbeit keinen Weg in den kleiner werdenden Raum geschützter Arbeitsmärkte zurückfinden.

Die Sozialstrukturanalyse konnte bislang vergleichsweise leicht nachweisen, welche Beschäftigtengruppen typischerweise besonderen Risiken ausgesetzt sind und deswegen zielgruppenspezifische Programme rechtfertigen. Von hoher Bedeutung ist jedoch, dass sich allein schon in den bekannten Segmenten der Existenz gefährdenden Lebensverläufe sowie bei Arbeitslosen unterschiedliche Brüche und vielschichtige Gefährdungsprofile zeigen, zugleich aber auch vielschichtige individuelle Bewältigungsprofile offenbaren. Diese Vielschichtigkeit wird von der Arbeitsmarkt- und Sozialpolitik bislang nur unzureichend berücksichtigt und genutzt.

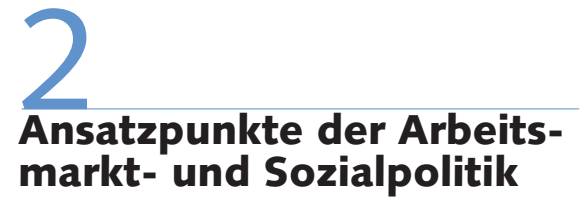

Grundsätzlich erzeugt Flexibilität ihrerseits Flexibilität und setzt zugleich aber Stabilität voraus. So erzeugen flexibilisierte Anforderungen am Arbeitsmarkt Anpassungszwänge aufseiten der Individuen. Diese können wiederum nur durch ein flexibles Verarbeitungsvermögen auf der Basis fundierter Handlungskompetenzen und stabiler Lebensarrangements bewältigt werden. Doch nicht alle Individuen sind in jeder Lebenssituation dazu in der Lage, die gestiegenen flexiblen Bewältigungsleistungen ohne gesicherte soziale Unterstützung zu erfüllen. Die Sozialpolitik ist daher damit konfrontiert, Einkommensrisiken bei kritischen Übergängen im Lebensverlauf abzusichern. Die aktive Arbeitsmarktpolitik muss sich in höherem Maße an den divergierenden Fähigkeiten und Möglichkeiten der Individuen ausrichten.

\subsection{GESTALTUNG KRITISCHER ÜBERGÄNGE IM LEBENSVERLAUF}

Wir haben festgestellt: Die bisherige Verbindung zwischen dem immer flexibleren Erwerbsarbeits- und den lohnarbeits- und „normalarbeits“-fixierten sozialen Sicherungssystemen löst sich zunehmend. Und so stellt sich die Frage, in welcher Weise eine sozialstaatliche Neuausrichtung die Bedürfnisse der Wirtschaft und Gesellschaft nach Anpassungsflexibilität und Risikobereitschaft mit dem Bedürfnis der Beschäftigten nach sozialer Absicherung bei Investitionen und Anpassungsversuchen verbinden kann. Erst in einer solchen Konstellation kann der Sozialstaat seine Funktion als Produktivkraft voll entfalten und zu einer Entlastung der angespannten Situation am Arbeitsmarkt und in den sozialen Sicherungssystemen beitragen.

Auf unsicherem moorigen Grund wird niemand weite Sprünge wagen. Und so gilt dann auch, dass soziale Sicherungssysteme, sofern sie Basissicherheiten bieten, in der Lage sind, innovatives Verhalten zu stärken. Das erst erleichtert den Akteuren Anpassungen an institutionelle und organisatorische Neuarrangements, seien es Neuorientierungen im Bildungssystem, in der Kinderbetreuung, der Alterssicherung, der Aktivierung in der Sozial- und Arbeits- 
marktpolitik oder im Erwerbsleben selbst. Versicherungen fördern also nicht nur Versuchungen zur „Mitnahme“ von Leistungen (obgleich diese zu berücksichtigen sind), sondern können ebenso die Bereitschaft zu Investitionen und riskanten Entscheidungen fördern, die sonst ausbleiben würden.

Heute bietet des deutsche Sozialsystem jedoch weder ausreichend Schutz gegen riskante und normale Brüche im Erwerbsverlauf noch offeriert es hinreichende Anreize für den Einzelnen, sich den veränderten Herausforderungen offensiv zu stellen. Dabei mangelt es vor allem an einer Absicherung gegen Risiken, die es den Menschen erlaubt, kritische Übergänge auch selbst zu wagen. Weitgehend unberücksichtig bleiben dabei zeitweilige Übergänge zwischen Vollzeit- und Teilzeitbeschäftigung sowie zwischen abhängiger Beschäftigung und Selbststständigkeit wie auch umgekehrt Übergänge in einen zweiten oder dritten Bildungsweg, Kombinationen von Arbeit und Bildung, flexible Übergänge zwischen Erwerbstätigkeit und Pflegezeiten von Angehörigen oder graduelle (anstelle abrupter) Übergänge in die Rentenphase. Hier zeigen sich wichtige Ansatzpunkte für die Weiterentwicklung von Maßnahmen, die sich dann zugleich auch bruchlos in nationale und EU-weite Vorschläge zu Flexicurity-Ansätzen einbinden lassen (Struck 2009).

Einen wichtigen Ansatzpunkt bietet hier die Weiterentwicklung der Arbeitslosenversicherung zu einer Beschäftigungsversicherung (Schmid 2008; Schuster 2005). Eine Beschäftigungsversicherung sichert das Einkommensrisiko nicht nur bei Arbeitslosigkeit, sondern auch bei kritischen Übergängen im Lebenslauf ab. Eine solche Weiterentwicklung des sozialen Sicherungssystems ließe sich sowohl finanzieren als auch gesellschaftlich legitimieren: Sie würde bestehende staatliche Transferleistungen wie Bafög, Arbeitslosengeld, Weiterbildungs- und Umschulungsprogramme, Familienförderung, Existenzgründerhilfen oder die soziale Grundsicherung etc. integrieren, womit sie (anders als Überlegungen zum bedingungslosen Grundeinkommen, siehe dazu Straubhaar 2008) realistisch und ohne Systemwechsel umsetzbar ist.

Dabei ist der Zugriff auf Sozialleistungen durch klare und gesellschaftlich akzeptierte Regeln zu gestalten. Auf eine hohe gesellschaftliche Akzeptanz dürften - neben dem Arbeitslosengeld und der sozialen Grundsicherung - vermutlich Kindererziehungszeiten und Phasen der Pflege von Angehörigen, (Weiter-)Bildungsphasen sowie Existenzgründungsphasen treffen. Der $\mathrm{Zu}$ griff auf Sozialleistungen bestünde nicht nur in einer zeitlich befristeten Übergangsfinanzierung gesellschaftlich anerkannter Reproduktions- und Innovationsphasen (etwa nach einer Mindestbeschäftigungszeit in einer Rahmenfrist), sondern würde auch die Anrechnungszeiten und Transferleistungen in der Alterssicherung (neben den bestehenden Erziehungszeiten etwa auch Ausgleiche bei Teilzeitbeschäftigung) und in stärkerem Maße als bislang (Weiter-)Bildungsphasen berücksichtigen. Bei Qualifizierungs- und Arbeitsförderungsmaßnahmen ist eine Verbesserung der Prognosesysteme und der Beratungskapazitäten der Arbeitsagenturen sowie zum Teil von der Industrie- und Handelskammer (IHK) und Bildungsinstitutionen etc. vorausgesetzt. Aufgrund der vielfach prekären Abfolge und Gleichzeitigkeit von Selbstständigkeit und abhängiger Erwerbsarbeit wären zudem auch Selbstständige in das Sozialversicherungssystem einzubeziehen. Und nicht zuletzt würde eine Stärkung lohnunabhängiger (d.h. stärker steuerfinanzierter) Sozialsysteme die Abgabenbelastung der Arbeitsverhältnisse verringern, Beschäftigungsspielräume erweitern und die Binnennachfrage nach Konsumgütern aufseiten der Beschäftigten erhöhen.

Die wichtigsten Vorteile einer solchen Strategie liegen erstens darin, dass sie das innovative Verhaltensrisiko von Arbeitnehmern und Arbeitgebern gleichermaßen stärkt. Die Neugestaltung zielt darauf, die Flexibilität der Arbeitszeiten im Lebenslauf, die zwischenbetriebliche Mobilität und die Weiterbildungsbereitschaft zu steigern. Sie bietet Beschäftigten Schutz vor Abstiegen bei Entlassungen, sie verbessert qualifikatorische und motivationale Ausgleichsprozesse auf Arbeitsmärkten vor allem durch eine effizientere Suche nach einem passgerechten Arbeitgeber. Darüber hinaus verbessert sie die Chancen, Arbeit und Familie miteinander zu vereinbaren.

Zweitens ermöglicht die soziale Absicherung von Flexibilitätsansprüchen auch Menschen in höheren Einkommens- und Qualifikationsgruppen, ihre Erwerbsarbeitszeiten temporär zu vermindern oder für eine gewisse Zeit aus der Erwerbsarbeit auszusteigen. Die hierdurch entstehenden Phasen der Nichtarbeit bieten Raum für die
Arbeitssuche. Das ist ein wichtiger Punkt, der bislang auch von Protagonisten einer verbesserten „Übergangssicherung“" (etwa Schmid 2008) zuwenig beachtet wird.

Eine entsprechende Zunahme der Arbeitsmarktmobilität setzt voraus, dass das Qualifizierungsniveau der bislang weitgehend chancenlosen Beschäftigtengruppen durch aktive Arbeitsmarktpolitik fortlaufend an die sich wandelnden Tätigkeitsanforderungen angepasst wird.

\subsection{BERÜCKSICHTIGUNG INDI- VIDUELLER LEBENSSITUATIONEN}

Die zuvor betrachtete, Innovation und Qualifikation fördernde Rahmensicherung von Übergängen richtet sich auf notwendige Anpassungen der finanziellen und infrastrukturellen Grundausstattung für Individuen, um den flexibleren Anforderungen einer Arbeitsgesellschaft gerecht zu werden. Damit Individuen diese Übergänge erfolgreich und flexibel gestalten können, sind zugleich jedoch soziale und kulturelle Kompetenzen, Reflexionsvermögen und Lernkompetenz bei Wahrung der zuvor erworbenen berufsrelevanten allgemeinen und z. T. spezifischen Fähigkeiten und Fertigkeiten erforderlich. Bei derartigen Kompetenzen handelt es sich um individuelle und kollektive Güter: Individuen steigern ihre selbstbestimmte, wenngleich an Erwerbsarbeit ausgerichtete Anpassungsflexibilität, womit zugleich eine wirksame Verbesserung der Strukturen am Arbeitsmarkt einhergeht.

Allerdings können viele Erwerbspersonen diese notwendigen Kompetenzen aufgrund mangelnder Gelegenheitsstrukturen, kritischer Lebensereignisse oder geringer Vorkenntnisse nicht aufbringen. Hinzu kommt, dass diese Kompetenzen auch im Rahmen einer Beschäftigung - und gerade auch in numerisch flexiblen Beschäftigungsformen wie Leiharbeit oder Befristungen ohne betriebliche Anschlussperspektive - vielfach nicht entwickelt und signalisiert werden können. Aus unseren Befragungen (Köhler et al. 2008, S. 31ff.) wissen wir, dass die überwiegende Zahl der Betriebe, die häufig auf externe Märkte für kurzfristige Beschäftigungsbeziehungen zurückgreift, selbst wenig zur Funktionsfähigkeit der externen Arbeitsmärkte beiträgt. Die kurzfristig ausgeübten Tätigkeiten bieten informell wenige und formell keine Qualifizierungsmöglichkeiten. $\mathrm{Zu}$ dem werden Vernetzungen für berufliche 
Anschlussperspektiven vonseiten des Betriebes nicht unterstützt. Die Arbeitstätigkeiten und -ergebnisse selbst weisen nur sehr allgemeine Merkmale auf, die einem potenziellen neuen Arbeitgeber keine Einblicke in die Fertigkeiten und Fähigkeiten des Arbeitsuchenden erlauben. Aus diesem Grund sind Kompetenzen zur Steigerung der Beschäftigungsfähigkeit auch im Rahmen der Arbeitsmarkt- und Sozialpolitik zu fördern. Die Frage ist auch hier: wie?

Bereits seit Beginn der 1990er Jahre lässt sich die Arbeitsmarkt- und Sozialpolitik durch das Primat der "Aktivierung“ zur Entwicklung individueller Handlungskompetenzen kennzeichnen. Ziel ist die Steigerung der Anpassungsflexibilität aller erwerbslosen, jedoch erwerbsfähigen Erwerbspersonen. Aktuell und explizit ist damit zunächst die Kategorie des „Forderns“ verbunden. Eine Universalisierung der Arbeitsmarktteilhabe als sozialpolitische Norm (Vobruba 1989) bedeutet, dass der Sozialstaat zur Erzielung arbeitsmarktkonformen Verhaltens der Erwerbstätigen auf geeignete Lohnanreize setzt bzw. dies über Mindestlohnregelungen beeinflusst oder den Nutzen der Nichtarbeit durch niedrige Transferleistungen mindert (Lohnabstandsgebot) sowie über Sanktionen und gegebenenfalls pädagogisierende Elemente versucht, die Arbeitsaufnahme zu steuern (Dahme/Wohlfahrt 2003). Dabei werden in Deutschland Transferleistungen und Fördermaßnahmen der staatlichen Arbeitsmarkt- und Sozialpolitik unmittelbarer als zuvor an überprüfte Aktivitäten der Transferempfänger gebunden. Über Änderung ihres Verhaltens sollen erwerbsfähige Transferbezieher ihre Integration in das Erwerbsleben erreichen. Maßnahmen sind Kürzungen sozialer Leistungstransfers in Verbindung mit einer Ausweitung der Arbeitsverpflichtung von erwerbsfähigen Leistungsbeziehern, eine Minderung des Berufs- und Statusschutzes sowie eine strenge Beaufsichtigung, Disziplin und Anpassung zur Arbeit.

Wenn es richtig ist, dass viele Erwerbspersonen die hierfür notwendigen Kompetenzen nicht aufbringen können, dann erfordert eine (Workfare-)Politik der Aktivierung und Befähigung bzw. die damit verbundene Universalisierung der Arbeitsmarktteilhabe, dass der aktivierende Staat ebenfalls seine Förderungsrolle ernst nimmt. Dies ist voraussetzungsvoll. Bezogen auf die Arbeitsmarktpolitik erfordert die Steigerung der Anpassungsflexibilität zur Ar- beitsmarktteilhabe für alle Bürger vor allem Maßnahmen der Kompetenzsteigerung und der Aus- und Weiterbildung wie auch der Beratung, Vermittlung sowie weitere, aufeinander abgestimmte infrastrukturelle Angebote für die jeweilige Zielgruppe wie Kinderbetreuung, Gesundheitsund psychosoziale Dienste oder Schuldnerberatung etc.

Betrachten wir nun wiederum die aktuelle Arbeitsmarkt- und Sozialpolitik, dann lässt sich feststellen, dass das Fördern eng auf die Eingliederung in Arbeit ausgerichtet ist („Matchingstrategie“). Wie bisherige Studien zeigen, verführt aber gerade die Matchingstrategie und damit die Konzentration auf schnelle Eingliederungserfolge und standardisierte Routinen (kontrolliert durch Monitoring der Behörden) in der Beratung und Vermittlung dazu, die biografisch erworbenen Fertigkeiten und Fähigkeiten sowie soziale und psychische Problemlagen der Arbeitsuchenden zu ignorieren. So werden auch Abbrüche von Maßnahmen oder schnelles Ausscheiden aus einer neuen Beschäftigung oder eine Nichtberücksichtigung eines offeriertes Maßnahmen- oder Stellenangebotes dann nicht selten als defizitäre Verhaltensweisen und Persönlichkeitsstrukturen sanktioniert (Beathge-Kinsky et al. 2006; Marston et al. 2005). Der Schritt zur Unterstellung, diese wiederum seien die wesentlichen Ursachen für soziale Ausgrenzungen, ist dann nicht mehr weit (Mead 1997).

Hinzu kommt, dass knappe Mittel für Maßnahmen und ein geringes Arbeitsplatzangebot den Beratern zusätzlich wenig Anreize bieten, um Informationen über individuelle Bedürfnisse zu erheben (Brodkin 1997) und sich um Kenntnisse über berufsrelevante Handlungskompetenzen zu bemühen, die in der schulischen und beruflichen Bildung, in vorherigen Tätigkeiten, in der Familie oder in ehrenamtlichen Aktivitäten erworben wurden und nicht in standardisierter zertifizierter Form vorliegen.

Wenn Lebens- und Berufsverläufe diskontinuierlich sind, Individuen vielschichtige soziale und psychische Problemlagen sowie differenzierte Qualifikations- und Kompetenzprofile aufweisen und der Arbeitsmarkt zugleich durch immer flexiblere und unvorhersehbare Tätigkeitsanforderungen charakterisiert ist, dann müssen auch in der Arbeitsmarkt- und Sozialberatung individuell zugeschnittene Vereinbarungen getroffen werden.
Hier richtet sich das Fördern dann umfassend auf das Arbeitsmarktverhalten, wobei berufsrelevante Handlungskompetenzen und soziale Kontexte des Betroffenen einbezogen werden („Fürsorgestrategie“). Wichtig ist dabei die Stärkung der intrinsischen Motivation zur Kooperation und Eigenleistung. Bekanntlich wird diese eher durch Partizipation als durch Fremdkontrolle (Kruglanski 1975), eher durch gemeinsame Zielvereinbarung als durch Zielsetzung gefördert (Frey/Osterloh 1997). In der Beratung und Vermittlung ist dann eine angemessene Professionalität, die sich nach Oevermann $(1996,2002)$ in einer einzelfallbezogenen Bearbeitung - im Gegensatz zu einer standardisierten Massenbearbeitung - ausdrückt, nur möglich durch Expertise, freie Wahl der Mittel und Autonomie im Umgang mit den Klienten.

Eine „fürsorgliche “ und zukunftsfähige Arbeitsmarkt- und Sozialpolitik, die insbesondere dem sogenannten „Beratungsund Betreuungsklientel“ chancenreiche Arbeitsmarktzugänge vermitteln kann, muss dann nicht nur ausreichende Qualifizierung fördernde Maßnahmen bereitstellen - gerahmt durch die in Abschnitt 2.1 dargestellten Maßnahmen einer Beschäftigungsversicherung. Systematisch zu entdecken, zu berücksichtigen und zu fördern sind zugleich auch:

- die individuellen berufbezogenen Stärken und Kompetenzen, wobei auch jene Kompetenzen einzubeziehen sind, die informell on-the-job oder in der Schule, Familie, Freizeit etc. erworben wurden;

- das Reflexionsvermögen, eigene Potenziale und Verhaltensweisen zu erkennen und sie realistisch mit neuen Zielen abzustimmen;

- die Lernkompetenz zur Selbstorganisation der beruflichen (Weiter-)Qualifizierung auf Basis der bislang erworbenen beruflichen Handlungskompetenzen;

- die Sicherstellung der Signalisierbarkeit der bislang (insbesondere auch informell) erworbenen Kompetenzen, u.a. durch eine geeignete Dokumentation von Leistungen und Werken;

- die selbstorganisierte Fähigkeit, sich für berufliche Neuanschlüsse zu vernetzen, zu informieren, Kontakte zu knüpfen etc.;

- die Entwicklung der Sensibilität und Kompetenz, sich in neue $-z$. T. fremdkulturelle bzw. milieuferne - Arbeitszusammenhänge und betriebliche Sozialstrukturen einzufinden. 
Auf diese Weise können diskontinuierliche Karriereverläufe und wechselnde Qualifikationsanforderungen unterstützt werden. Arbeitgeber achten sehr genau auf die Passfähigkeiten der „harten Fakten“ zur beruflichen Qualifikation. Sie achten aber ebenso auf die sogenannten ,weichen Faktoren “ wie Zuverlässigkeit, Kooperationsfähigkeit, Analyse- und Reflexionsvermögen, Lernund Leistungsbereitschaft etc. Die Arbeitsvermittlung und Beratung sollte dies ebenfalls sehr bewusst tun, um Arbeitslosen und prekär Beschäftigten Übergänge in eine flexiblere Arbeitswelt neu zu ermöglichen.

\section{Fazit}

Arbeitsmärkte sind durch eine Zunahme an Flexibilität und Mobilität gekennzeichnet. Inwieweit sich hieraus Chancen oder Risiken ergeben, ist nicht zuletzt von der Ausgestaltung der Sozial- und Arbeitsmarktpolitik abhängig. Von besonderer Bedeutung ist die Gestaltung von Übergängen zwischen Phasen der Erwerbs- und Nichterwerbsarbeit.

Ein zentraler Ansatzpunkt besteht darin, Zugangsbarrieren zum ersten Arbeitsmarkt abzubauen und Phasen der Nichterwerbstätigkeit in stärkerem Maße als bisher sozial zu sichern. Dazu gehören eine vergleichsweise großzügige Einkommenssicherung bei Übergangsarbeitslosigkeit, ein hoher Arbeitskräfteumschlag, sowohl getragen durch eine starke Wirtschaftsdynamik als auch durch sozial gesicherte Ausstiegsphasen im Rahmen rechtlich gesicherter Gelegenheitsstrukturen, sowie das Recht auf Arbeitszeitreduzierung, auf Erziehungs- und Pflegezeiten oder auf Weiterbildung und nachholende Bildungsabschlüsse im Erwachsenenalter. So würde es einer an Sicherung von Übergängen orientierten Arbeitsmarkt- und Sozialpolitik angemessener gelingen, die gesellschaftlichen Reproduktionsnotwendigkeiten sicherzustellen.

Dabei erhalten auch Arbeitsuchende neue Chancen am ersten Arbeitsmarkt. Voraussetzung dafür ist dann allerdings, dass sich die Arbeitsvermittlung und -be- ratung deutlicher ihrer Verantwortung stellt, Übergänge zwischen verschiedenen Erwerbs- und Lebenspassagen zu ermöglichen. Und deutlicher orientieren sollte sie sich auch an den biografisch sehr spezifisch entwickelten Fähigkeiten und Erfahrungen, die Arbeitsuchende in ihrer Arbeitsbiografie und Lebenswelt erworben haben.

Aktuell Beschäftigte würden so vor Lohndumping und übergebührlichen Flexibilitäts- und Leistungsanforderungen geschützt. Polarisierungen zwischen (noch) geschützt Beschäftigten, prekär Beschäftigten und Arbeitslosen würden gemindert.

Auf kurzfristige Sicht mag sich eine solche Ausrichtung einer stark an unmittelbarer Kostenminimierung orientierten Arbeitsmarkt- und Sozialpolitik entziehen. Mittelfristig erfolgreicher ist jedoch eine Strategie, die sich an gesellschaftlichen Reproduktionsnotwendigkeiten orientiert und zukunftsfähige Investitionen und neue Freiheiten in Arbeit, Bildung, Familie und Freizeit ermöglicht.

\section{LITERATUR}

Alda, H. (2006): Sekundäre Arbeitsmarktintegration als Beobachtungskonzept sozioökonomischer Berichterstattung, in: SOEB (Hrsg): Berichterstattung zur sozioökonomischen Entwicklung Deutschlands, Zweiter Bericht - Zwischenbericht Teil I, Göttingen, S. 164-167

Allmendinger, J. (1994): Lebensverlauf und Sozialpolitik. Die Ungleichheit von Mann und Frau und ihr öffentlicher Ertrag, Frankfurt a. M./ New York

Baethge-Kinsky, V./Bartelheimer, P./Henke, J./Land, R./Willisch, A./ Wolf, A. (2006): Neue soziale Dienstleistungen nach SGB II (Konzeptstudie), Forschungsbericht, Göttingen

Beck, U./Beck-Gernsheim E. (1993): Nicht Autonomie, sondern Bastelbiographie, in: Zeitschrift für Soziologie 3, S. 178-187

Brodkin, E. Z. (1997): Inside the Welfare Contract: Discretion and Accountability in State Welfare Administration, in: Social Service Review 3, S. 1-33

Bundesagentur für Arbeit (2008): Branchen und Berufe in Deutschland Zeitarbeit, Nürnberg
Dahme, H.-J./Wohlfahrt, N. (2003): Aktivierungspolitik und der Umbau des Sozialstaates. Gesellschaftliche Modernisierung durch angebotsorientierte Sozialpolitik, in: Dies. (Hrsg.): Soziale Arbeit für den aktivierenden Staat, Opladen, S. 75-102

Frey, B. S./Osterloh, M. (1997): Sanktionen oder Seelenmassage? Motivationale Grundlagen der Unternehmensführung, in: Die Betriebswirtschaft 4, S. 307-321

Kalina, T./Weinkopf, C. (2009): Niedriglohnbeschäftigung 2007 weiter gestigen - zunehmende Bedeutung von Niedrigstlöhnen, IAQ-Report 2009-05, Essen

Kaufmann, F.-X. (1986): Steuerungsprobleme der Sozialpolitik, in: Heinze, R. G. (Hrsg.): Neue Subsidarität: Leitidee für eine zukünftige Sozialpolitik?, Opladen, S. 39-63

Köhler, C./Struck, O./Grother, M./Krause, A./Krause, I./Schröder, T. (2008): Offene und geschlossene Beschäftigungssysteme. Determinanten, Risiken, Nebenwirkungen, Wiesbaden 
Kruglanski, A. W. (1975): The endogenous-exogenous partition in attribution theory, in: Psychological Review 6, S. 387-406

Marston, G./ Larsen, J. E./McDonald, C. (2005): The Active Subjects of Welfare Reform: a Street-Level Comparison of Employment Services in Australia and Denmark, in: Social Work \& Society 2, S. 141-157

Mead, L. M. (Hrsg.) (1997): The new paternalism. Supervisory approaches to poverty, Washington D. C.

Mückenberger, U. (1985): Die Krise des Normalarbeitsverhältnisses. Hat das Arbeitsrecht noch Zukunft?, in: Zeitschrift für Sozialreform 7, S. 415434 und 8, S. 457-475

Oevermann, U. (1996): Theoretische Skizze einer revidierten Theorie professionalisierten Handelns, in: Combe, A./Helsper, W. (Hrsg.): Pädagogische Professionalität, Frankfurt a. M., S. 70-182

Oevermann, U. (2002): Professionalisierungsbedürftigkeit und Professionalisiertheit pädagogischen Handelns, in: Kraul, M. (Hrsg.): Biographie und Profession, Bad Heilbrunn, S. 119-163

Sachverständigenrat zur Begutachtung der gesamtwirtschaftlichen Entwicklung (SVR) (2007): Das Erreichte nicht verspielen, Jahresgutachten 2007/08, Wiesbaden

Schmid, G. (2008): Von der Arbeitslosen- zur Beschäftigungsversicherung. Wege zu einer neuen Balance individueller Verantwortung und Solidarität durch eine lebenslauforientierte Arbeitsmarktpolitik, Gutachten für die Friedrich-Ebert-Stiftung, Bonn

Schuster, J. (2005): Beschäftigungsversicherung - Die Antwort auf sozialstaatliche Herausforderungen der Arbeitsgesellschaft, in: Zeitschrift für sozialistische Politik und Wirtschaft 143, S. 20-22
Sengenberger, W. (1987): Struktur und Funktionsweise von Arbeitsmärkten. Die Bundesrepublik Deutschland im Vergleich, Frankfurt a. M./ New York

Straubhaar T. (Hrsg.) (2008): Bedingungsloses Grundeinkommen und Solidarisches Bürgergeld - mehr als sozialutopische Konzepte, Hamburg Struck, O. (2006): Flexibilität und Sicherheit. Empirische Befunde, theoretische Konzepte und institutionelle Gestaltung von Beschäftigungsstabilität, Wiesbaden

Struck, O. (2009): Europäische Flexicurity - Eine Leitidee im Fokus einer Theorie gesellschaftlichen Wandels, in: Eigmüller M./Mau, S. (Hrsg.): Gesellschaftstheorie und Europapolitik. Sozialwissenschaftliche Ansätze zur Europaforschung, Wiesbaden, im Erscheinen

Struck, O./Grotheer, M./Schröder,T./Köhler, C. (2007): Instabile Beschäftigung. Neue Ergebnisse zu einer alten Kontroverse, in: Kölner Zeitschrift für Soziologie und Sozialpsychologie 2, S. 294-317

Vobruba, G. (1989): Arbeit und Essen. Politik an den Grenzen des Arbeitsmarktes, Wien

Vobruba, G. (1990): Lohnarbeitszentrierte Sozialpolitik in der Krise der Lohnarbeit, in: Ders. (Hrsg.): Strukturwandel der Sozialpolitik, Frankfurt a. M., S. 11-80

Williamson, O. E./Wachter, M. L./Harris, J. E. (1975): Understanding the Employment Relation: Analysis of Idiosyncratic Exchange, in: Bell Journal of Economics 1, S. 250-278 